\title{
ERGODIC GROUP ACTIONS WITH NONUNIQUE INVARIANT MEANS
}

\author{
CHING CHOU
}

\begin{abstract}
Let $M(X, G)$ be the set of $G$-invariant means on $L^{\infty}(X, B, P)$, where $G$ is a countable group acting ergodically as measure preserving transformations on a nonatomic probability space $(X, B, P)$. We show that if there exists $\mu \in M(X, G), \mu \neq P$, then $M(X, G)$ contains an isometric copy of $\beta N \backslash N$, where $\beta N \backslash N$ is considered as a subset of $\left(l^{\infty}\right)^{*}$. This provides an answer to a question raised by J. Rosenblatt in 1981 .
\end{abstract}

Let $(X, B, P)$ be a nonatomic probability space, $G$ a countable group, and $(g, x) \rightarrow g x$ a measure preserving ergodic action of $G$ on $(X, B, P)$. Then $G$ also acts on $L^{\infty}(X)=L^{\infty}(X, B, P):(g \cdot f)(x)=f\left(g^{-1} x\right), f \in L^{\infty}(X), g \in G, x \in X$. A positive linear functional of norm 1 on $L^{\infty}(X)$ is called a mean. A mean $m$ is said to be $G$-invariant if $m(g \cdot f)=m(f)$ for $g \in G$ and $f \in L^{\infty}(X)$. We will denote the set of $G$-invariant means on $L^{\infty}(X)$ by $M(X, G)$. If we consider $P$ as the functional on $L^{\infty}(X)$ that sends $f$ to $\int f d P$, then $P \in M(X, G)$.

It is natural to ask under what conditions will $P$ be the unique $G$-invariant mean on $L^{\infty}(X)$. This problem was first studied by del Junco and Rosenblatt [3]. They proved that if $G$ is amenable then $M(X, G) \supsetneqq\{P\}$ by showing that $X$ contains small almost invariant sets. The existence of almost invariant sets was also studied by Schmidt [10]. These two papers inspired further investigations of $G$-invariant means by Rosenblatt [9], Schmidt [11], and Losert and Rindler [6], among others.

In this short note we will only address a question raised by Rosenblatt in [9]: If $G$ is amenable then $M(X, G) \supsetneqq\{P\}$; what can be said about the cardinality of $M(X, G)$ ? We will show that whenever $M(X, G)$ is not a singleton (in particular, when $G$ is amenable) then card $M(X, G) \geq 2^{c}$, where $c$ is the cardinality of the continuum. To prove our result, we will need the following fundamental result of Rosenblatt [9, Theorem 1.4]; see also [3].

THEOREM A (ROSENBLATT [9]). $M(X, G) \supsetneqq\{P\}$ if and only if there exists a sequence of measurable sets $\left\{A_{n}\right\}$ in $X$ such that

(i) $P\left(A_{n}\right)>0, \lim _{n} P\left(A_{n}\right)=0$,

(ii) for each $g \in G, \lim _{n} P\left(A_{n} \Delta g A_{n}\right) / P\left(A_{n}\right)=0$.

A sequence $\left\{A_{n}\right\}$ that satisfies (i) and (ii) is called an arbitrarily small asymptotically invariant sequence in [9].

Received by the editors April 21, 1986.

1980 Mathematics Subject Classification. Primary 43A07, 28D15.

Key words and phrases. Invariant means, ergodic group actions, asymptotically invariant sequences.

(C) 1987 American Mathematical Society $0002-9939 / 87 \$ 1.00+\$ .25$ per page 
Let $\mathcal{F}=\left\{\theta \in\left(l^{\infty}\right)^{*}: \theta \geq 0,\|\theta\|=1\right.$, and $\theta\left(\left(t_{n}\right)\right)=0$ whenever $\left(t_{n}\right) \in l^{\infty}$ and $\left.\lim _{n} t_{n}=0\right\}$. Let $N$ be the set of positive integers with discrete topology and $\beta N$ its Stone-Čech compactification. Then $\beta N \backslash N$ can be considered as a subset of $\mathcal{F}$, and consequently, card $\mathcal{F}=2^{c}$. (In fact, the $w^{*}$-closed convex hull of $\beta N \backslash N$ is $\mathcal{F}$.) We are now ready to state and prove our main result.

THEOREM. Let $G$ be a countable group and let $(g, x) \rightarrow g x$ be a measure preserving ergodic action of $G$ on a nonatomic probability space $(X, B, P)$. If $M(X, G) \supsetneqq$ $\{P\}$ then there exists a linear isometry $\Lambda$ of $\left(l^{\infty}\right)^{*}$ into $L^{\infty}(X)^{*}$ such that $\Lambda(\mathcal{F}) \subset$ $M(X, G)$; in particular, $\operatorname{card} M(X, G) \geq 2^{c}$.

ProOF. By Theorem A, there exists a sequence of measurable sets $\left\{A_{n}\right\}$ in $X$ which satisfies conditions (i) and (ii). By taking a subsequence, if necessary, we may replace (i) by

$$
0<P\left(A_{n+1}\right)<\frac{1}{2^{n}} P\left(A_{n}\right), \quad n=1,2, \ldots .
$$

Write $A_{n}=B_{n} \cup C_{n}$ where $B_{n}=A_{n} \backslash\left(A_{n+1} \cup A_{n+2} \cup \cdots\right)$ and $C_{n}=A_{n} \cap\left(A_{n+1} \cup\right.$ $\left.A_{n+2} \cup \cdots\right)$. Then, by (i)',

$$
\begin{aligned}
P\left(C_{n}\right) & \leq \sum_{j=n+1}^{\infty} P\left(A_{j}\right) \leq \frac{1}{2^{n}} P\left(A_{n}\right)+\frac{1}{2^{n+1}} P\left(A_{n+1}\right)+\cdots \\
& <\frac{1}{2^{n}} P\left(A_{n}\right)\left(1+\frac{1}{2}+\frac{1}{2^{2}}+\cdots\right)=\frac{1}{2^{n-1}} P\left(A_{n}\right) .
\end{aligned}
$$

If $g \in G$, then $B_{n} \backslash g B_{n} \subset\left(A_{n} \backslash g A_{n}\right) \cup g C_{n}$, and hence

$$
\frac{P\left(B_{n} \backslash g B_{n}\right)}{P\left(B_{n}\right)} \leq\left(\frac{P\left(A_{n} \backslash g A_{n}\right)}{P\left(A_{n}\right)}+\frac{P\left(g C_{n}\right)}{P\left(A_{n}\right)}\right) \frac{P\left(A_{n}\right)}{P\left(B_{n}\right)} .
$$

Note that by (ii), $P\left(A_{n} \backslash g A_{n}\right) / P\left(A_{n}\right) \rightarrow 0$, and by (iii),

$$
P\left(g C_{n}\right) / P\left(A_{n}\right)=P\left(C_{n}\right) / P\left(A_{n}\right) \leq 1 / 2^{n-1} \rightarrow 0
$$

and

$$
P\left(A_{n}\right) / P\left(B_{n}\right)=P\left(A_{n}\right) /\left(P\left(A_{n}\right)-P\left(C_{n}\right)\right) \leq 1 /\left(1-1 / 2^{n-1}\right) \rightarrow 1 .
$$

So $P\left(B_{n} \backslash g B_{n}\right) / P\left(B_{n}\right) \rightarrow 0$. Therefore, we have constructed a sequence of measurable sets $\left\{B_{n}\right\}$ in $X$ such that

(a) $P\left(B_{n}\right)>0, \lim _{n} P\left(B_{n}\right)=0$,

(b) $\lim _{n} P\left(B_{n} \Delta g B_{n}\right) / P\left(B_{n}\right)=0$ for $g \in G$,

(c) $B_{n} \cap B_{k}=\varnothing$ if $n \neq k$.

Proceed now as in the proof of Theorem 3.3 of [2]. Let $\pi: L^{\infty}(X) \rightarrow l^{\infty}$ be defined by $(\pi f)(n)=\left(1 / P\left(B_{n}\right)\right) \int_{B_{n}} f d P$. It is easily checked that $\pi$ is linear, $\|\pi\|=1$, and $\pi \geq 0$. Given $\left(t_{n}\right) \in l^{\infty}$, let $f=\sum t_{n} \chi_{B_{n}}$. Then, by (c), $\pi f=\left(t_{n}\right)$. Therefore $\pi$ is onto and hence $\pi^{*}$ is an isometry. To see that $\pi^{*}$ is the isometry that we are looking for, it remains to show that if $\theta \in \mathcal{F}$ then $\pi^{*} \theta \in M(X, G)$. Indeed, if $\theta \in \mathcal{F}, g \in G$, and $f \in L^{\infty}(X)$, then

$$
\begin{aligned}
|\pi(f-g \cdot f)(n)| & =\left|\frac{1}{P\left(B_{n}\right)}\left(\int_{B_{n}} f d P-\int_{B_{n}}(g \cdot f) d P\right)\right| \\
& \leq\|f\|_{\infty} \frac{P\left(B_{n} \Delta g B_{n}\right)}{P\left(B_{n}\right)} \rightarrow 0, \text { as } n \rightarrow \infty, \text { by (b). }
\end{aligned}
$$


Since $\theta \in \mathcal{F}, \theta(\pi(f-g \cdot f))=0$ or $\pi^{*} \theta(f)=\pi^{*} \theta(g \cdot f)$. Thus $\pi^{*} \theta \in M(X, G)$ and the proof is complete.

COROLLARY. Suppose that $G$ is a countable amenable group acting ergodically as measure preserving transformations on a nonatomic probability space $(X, B, P)$. Then $\operatorname{card} M(X, G) \geq 2^{c}$.

ProOF. By Theorem 2.1 of $[9]$, card $M(X, G) \geq 2$ and hence by the above theorem, $\operatorname{card} M(X, G) \geq 2^{c}$.

REMARKS. (1) We have considered similar isometric embeddings of $\mathcal{F}$ in [1 and 2]. In particular, we could have quoted Theorem 2.4 of [2] to shorten the proof of our theorem here. Since the proof of Theorem 2.4 is quite involved, we prefer the direct construction of $\left\{B_{n}\right\}$ as given above. Recently, Granirer [5] has studied embeddings of $₹$ that are not necessarily isometric, in more general settings, by applying $\mathrm{H}$. Rosenthal's fundamental canonical $l^{1}$-basis theorem.

(2) It is known that a countable group $G$ has Kazhdan's Property $\mathrm{T}$ if and only if $L^{\infty}(X)$ always has a unique $G$-invariant mean whenever $G$ acts ergodically as measure preserving transformations on nonatomic probability space $(X, B, P)$ and $G$ is amenable if and only if no such actions of $G$ admit unique $G$-invariant means (see Schmidt [11]). The group $\mathrm{SL}(2, \mathbf{Z})$ is neither amenable nor does it have property $\mathbf{T}$. Schmidt [10] showed that the natural action of $\operatorname{SL}(2, \mathbf{Z})$ on $\mathbf{T}^{2}$ has no arbitrarily small asymptotically invariant sequences and hence $L^{\infty}\left(\mathbf{T}^{2}\right)$ has a unique $\operatorname{SL}(2, \mathbf{Z})$-invariant mean (see also [6 and $\mathbf{9}]$ ). On the other hand, an explicit ergodic action of $\mathrm{SL}(2, \mathbf{Z})$ that admits more than one $G$-invariant mean is described in [11]. By our theorem, this action admits at least $2^{c} G$-invariant means.

(3) Using Theorem A, Margulis [7] and Sullivan [12] proved independently that, for $n \geq 4$, any $\mathrm{SO}(n+1)$-invariant mean on $L^{\infty}\left(S^{n}, \mathcal{M}, m_{n}\right)$ is proportional to the Lebesgue measure $m_{n}$. ( $\mathcal{M}$ is the $\sigma$-algebra of Lebesgue measurable subsets of $S^{n}$.) More recently, Drinfel'd [4] was able to extend their result to the cases $n=2$ and 3. Thus the Banach-Ruziewicz problem for $S^{n}$ has been completely solved. The corresponding Banach-Ruziewicz problem for $\mathbf{R}^{n}, n \geq 3$, was solved by Margulis [8].

\section{REFERENCES}

1. C. Chou, On topologically invariant means on a locally compact group, Trans. Amer. Math. Soc. 151 (1970), 443-456.

2. Topological invariant means on the von Neumann algebra $V N(G)$, Trans. Amer. Math. Soc. 273 (1982), 207-229.

3. A. del Junco and J. Rosenblatt, Counterexamples in ergodic theory and number theory, Math. Ann. 245 (1979), 185-197.

4. V. G. Drinfel'd, Finitely additive measures on $S^{2}$ and $S^{3}$, invariant with respect to rotations, Functional Anal. Appl. 18 (1985), 245-246.

5. E. E. Granirer, Geometric and topological properties of certain $w^{*}$ compact convex subsets of double duals of Banach spaces, which arise from the study of invariant means, Illinois J. Math. (to appear).

6. V. Losert and H. Rindler, Almost invariant sets, Bull. London Math. Soc. 13 (1981), 145-148.

7. G. A. Margulis, Some remarks on invariant means, Monatsh. Math. 90 (1980), 233-235.

8. __ Finitely-additive invariant measures on Euclidean spaces, Ergodic Theory Dynamical Systems 2 (1982), 383-396.

9. J. Rosenblatt, Uniqueness of invariant means for measure preserving transformations, Trans. Amer. Math. Soc. 265 (1981), 623-636. 
10. K. Schmidt, Asymptotically invariant sequences and an action of $\mathrm{SL}(2, \mathbf{Z})$ on the 2 -sphere, Israel J. Math. 37 (1980), 193-208.

11. - Amenability, Kazhdan's property $\mathrm{T}$, strong ergodicity and invariant means for ergodic actions, Ergodic Theory Dynamical Systems 1 (1981), 223-236.

12. D. Sullivan, For $n>3$ there is only one finitely-additive rotationally invariant measure on the $n$-sphere defined on all Lebesgue measurable sets, Bull. Amer. Math. Soc. (N.S.) 1 (1981), 121-123.

Department of Mathematics, State University of NeW York at Buffalo, BUFFALO, NEW YORK 14214 